\title{
A procedure to join the force and volume ensemble statistical descriptions of granular media
}

\author{
Juan Sebastián Rey ${ }^{1, *}$, Jose Daniel Muñoz ${ }^{1, * *}$, and William Fernando Oquendo ${ }^{1, * * *}$ \\ ${ }^{1}$ Simulation of Physical Systems Group, Department of Physics \\ Universidad Nacional de Colombia - Carrera 30 No. 45-03, Ed. 404, Of. 348, Bogota D.C., Colombia
}

\begin{abstract}
Granular media consist of a large number of discrete particles interacting mostly through contact forces that, being dissipative, jeopardizes a classical statistical equilibrium approach based on energy. Instead, two independent equilibrium statistical descriptions have been proposed: the Volume Ensemble and the Force Network Ensemble. Hereby, we propose a procedure to join them into a single description, using Discrete Element simulations of a granular medium of monodisperse spheres in the limit state of isotropic compression as testing ground. By classifying grains according to the number of faces of the Voronoï cells around them, our analysis establishes an empirical relationship between that number of faces and the number of contacts on the grain. In addition, a linear relationship between the number of faces of each Voronoï cell and the number of elementary cells proposed by T. Aste and T. Di Matteo in 2007 is found. From those two relations, an expression for the total entropy (volumes plus forces) is written in terms of the contact number, an entropy that, when maximized, gives an equation of state connecting angoricity (the temperature-like variable for the force network ensemble) and compactivity (the temperature-like variable for the volume ensemble). So, the procedure establishes a microscopic connection between geometry and mechanics and, constitutes a further step towards building a complete statistical theory for granular media in equilibrium.
\end{abstract}

\section{Introduction}

Granular media, like sand, coffee grains or mineral rocks, consist of a large number of discrete particles interacting mostly through contact forces [1]. Since their macroscopic properties arise from microscopic interactions, they should be perfect systems for applying statistical mechanics; however, the interactions are dissipative, which makes them hard to describe by using energy and temperature as main variables [2]. Instead, two main statistical equilibrium approaches have been proposed: the Edwards' Volume Ensemble [3, 4], which accounts for all grains' configurations in mechanical equilibrium inside a given volume, and the Force Network Ensemble, developed by Snoeijer, Tighe and coworkers[5-7], which takes into account all sets of contact forces in mechanical equilibrium with a given external stress. Nevertheless, a relationship between the compactivity $\chi$ and the angoricity $\alpha^{-1}$ (i.e. the temperature-like variables for those ensembles) has not been established.

Hereby, we propose how those two ensembles could be combined for the limit state of isotropic compression of a set of monodisperse spheres. This is done by looking at the statistical distribution of volumes for Voronoï cells with the same number of faces $s$ and the statistical distribution of pressures for grains with the same number

\footnotetext{
*e-mail: jsreyl@unal.edu.co

**e-mail: jdmunozc@unal.edu.co

***e-mail: woquendop@unal.edu.co
}

A video is available at https://doi.org/10.48448/0m24-tj43 of contacts $z$. Next, an empirical relationship connecting $s$ and $z$ is established that allows to find a connection between $\chi$ and $\alpha^{-1}$ by minimizing the total entropy against the coordination number $z$.

\section{Aste and Di Mateo's volume ensemble}

Edwards' Volume Ensemble takes the volume as the main thermodynamic variable, with an entropy accounting for all possible geometric configurations inside a total volume $V_{T}$ obeying volume exclusion and mechanical stability with external constraints, i.e. all possible jammed states $[4,8]$. In a illuminating proposal by Aste and Di Mateo [9] the volume $V_{T}$ is divided into $C$ statistically independent elementary cells of volume $v_{i}>v_{\min }$, where $v_{\text {min }}$ is a minimum volume due to steric exclusion. Because in isotropic compression the shape of such elementary cells should not be relevant, their volumes will be the degrees of freedom (DOF) for the system. Thus, we can define a phase space of $C$ dimensions where each coordinate is the excess volume $v_{i}^{\prime}=v_{i}-v_{\min }$ for each elementary cell. Then, it is possible to derive $[9,10]$ that the probability for a singe elementary volume to take the specific value $v$ is

$$
p(v)=\frac{1}{\langle v\rangle-v_{\min }} \exp \left(\frac{v-v_{\text {min }}}{\langle v\rangle-v_{\min }}\right),
$$

where $\langle v\rangle=V_{T} / C$ is the average volume per cell. So, the entropy for a single cell can be calculated as $S_{1}=1+$ 
$\ln \left(\frac{\langle v\rangle-v_{\min }}{\Lambda^{3}}\right)$; and since the entropy is an extensive quantity, the entropy for all $\mathrm{C}$ independent cells is

$$
S_{C}=C\left[1+\ln \left(\frac{\langle v\rangle-v_{\min }}{\Lambda^{3}}\right)\right],
$$

where $\Lambda$ is a reference length ${ }^{1}$. The temperature-like variable $\frac{1}{\chi}=\frac{\partial S}{\partial V_{T}} \Rightarrow \chi=\frac{V_{T}}{C}-v_{\min }$ coincides with Edward's compactivity and indicates how much free volume is there per elementary cell. Although we don't know for sure what those elementary cells are, by performing a Voronoï tessellation and assuming that each tessellation cell contains approximately the same number of $k_{v}$ elementary cells, it follows that Voronoï cells volumes $V_{\text {voro }}$ should exhibit a $k$-gamma distribution [11],

$$
P\left(V_{\text {voro }}\right)=\frac{1}{\Gamma\left(k_{v}\right) \chi^{k_{v}}}\left(V_{\text {voro }}-V_{\min }\right)^{k_{v}-1} e^{-\frac{V_{\text {voro }}-V_{\min }}{\chi}},
$$

with $V_{\min }=k_{v} v_{\min }$, a distribution that has been found in many experimental and computational experiments [12, 13], (with $k_{v} \approx 12$ for monodisperse cells in the limit state of isotropic compression [10]). This way, the average $\left\langle V_{\text {voro }}\right\rangle$ and the variance $\sigma_{V}^{2}$ of that distribution can be used to calculate the compactivity of the system $\chi$ as well as the number of elementary cells per tessellation cell $k_{v}$,

$$
\chi=\frac{\sigma_{V}^{2}}{\left\langle V_{\mathrm{voro}}\right\rangle-V_{\min }}, \quad k_{v}=\frac{\left(\left\langle V_{\mathrm{voro}}\right\rangle-V_{\min }\right)^{2}}{\sigma_{V}^{2}} .
$$

Furthermore, Oquendo et. al [10] derived a state equation between the compactivity and the packing fraction $\phi$ for the system

$$
\chi=A\left(\frac{1}{\phi}-\frac{V_{\text {min }}}{v_{\text {grain }}}\right) .
$$

which is satisfied with $A \approx 0.04481 d^{3}$ for the limit state of isotropic compression and $V_{\min } / v_{\text {grain }} \approx 1.3250$ for monodisperse spherical packings[14, 15].

\section{Force network ensemble}

A second major approach to the statistical mechanics granular media is the Force Network Ensemble (FNE)[5-7], which is based on the hyperstaticity of dense states, i.e., that the number of contacts per grain $z$ is larger than the one $z_{\text {iso }}$ necessary to solve the equilibrium and external stress constraints uniquely and, therefore, there are multiple valid force networks $\vec{f}$ for the system. The number of degrees of freedom for the contact force variables is $k_{f}=\frac{d_{f} N}{2}\left(\langle z\rangle-z_{\text {iso }}\right)$, with $d_{f}=1\left(d_{f}=d\right.$, the system's dimension) for frictionless (frictional) particles.

In the canonical ensemble under isotropic compression at pressure $p$ (that is, when the stress tensor is $\sigma_{\alpha \beta}=$ $p \delta_{\alpha \beta}$ ), each force network appears with a probability

$$
P(\vec{f})=\frac{e^{-\alpha p(\vec{f})}}{Z} \quad, \quad Z=\int_{0}^{\infty} d p \Omega(p) \exp (-\alpha p),
$$

${ }^{1}$ We use $\Lambda=\sqrt[3]{e \chi \mathrm{RCP}} \approx 0.301 d$ (from the Random Close Packing (RCP) [10]) where $\alpha^{-1}$ is the angoricity [3] and $\Omega(p)$ is the number of force networks fulfilling those constraints. Since $\Omega(p)$ indicates the content of the solution space spanning $k_{f}$ dimensions and $p$ can be considered as the typical diameter of one dimension [5], then $\Omega(p) \propto p^{k_{f}}$, and the following equation of state fulfills:

$$
\alpha\langle p\rangle=k_{f} .
$$

The FNE has been widely studied by theoretical, experimental and computational works [16-18]. In a recent Monte Carlo study of the FNE on a single grain at constant angoricity, Cardenas et. al. [19] found that the pressure per grain, calculated as the sum of the normal forces per particle $p_{i}=\sum_{j=1}^{z_{i}}\left|\vec{F}_{i j}^{n}\right|$, exhibits a k-gamma probability distribution,

$$
P(p)=\frac{1}{\Gamma(k) \theta^{k}} p^{k-1} e^{-\frac{p}{\theta}},
$$

with

$$
k=\frac{\langle p\rangle^{2}}{\sigma_{p}^{2}}, \quad \theta=\frac{\sigma_{p}^{2}}{\langle p\rangle}=\alpha^{-1},
$$

where $k \rightarrow k_{f}$ for low angoricities $\left(\alpha^{-1}<10^{-2}\right)$, satisfying the equation of state (7). This result suggests that the pressure can be considered as the sum of $k_{f}$ independent force variables $f_{i}$ with exponential distribution $P\left(f_{i}\right)=\alpha e^{-\alpha f_{i}}$. Thus, the entropy for one force variable will be $S_{1}^{f}=1+\ln \left(\frac{\alpha^{-1}}{p_{0}}\right)$, with $p_{0}$ a reference pressure ${ }^{2}$, and the total FNE entropy would be

$$
S_{k_{f}}^{f}=k_{f}\left[1+\ln \left(\frac{\alpha^{-1}}{p_{0}}\right)\right] .
$$

\section{Our work}

To study a possible connection between the pressure per grain and the volume of the Voronoï cells in the limit state of isotropic compression we performed DEM simulations with the software LIGGGHTS [20-22] on a 3D cubic packing of 80000 monodisperse spheres. The contact forces are Hertzian plus a dissipative term as in Ref. [20], with time step $\Delta t=10^{-5} \mathrm{~s}$. The limit configurations are obtained through four sequential procedures : A random insertion of particles inside a cubic box with initial length $L_{i}=0.3 m$ to create a dilute granular media with packing fraction $\phi \approx 0.2$, a particle grow for $t_{\text {grow }}=0.5 \mathrm{~s}$ until $\phi \approx 0.3$ in a process analogue to the LubachevskyStillinger's algorithm [23], a relaxation for $t_{\text {relax }}=0.2 \mathrm{~s}$ until the particles take fixed positions and their kinetic energy is dissipated and an isotropic compression of the walls for $t_{\text {compress }}=6 \mathrm{~s}$ with inertial number $I=\dot{\epsilon} d \sqrt{\frac{\rho_{m}}{P_{\text {wall }}}}=$ $5 \times 10^{-4}$ (where $\dot{\epsilon}$ is the deformation ratio, $d$ the particle diameter, $\rho_{m}$ the mass density of the system and $P_{\text {wall }}$ the external pressure applied on all walls). The simulation was run five times: one for each external pressure $P_{\text {wall }}=13.9,27.8,55.5,111.1$ and $222.2 \mathrm{~Pa}$, and the wall velocity $v_{\text {wall }}$ was set to satisfy the low inertial number.

\footnotetext{
${ }^{2}$ We choose $p_{0}=e \alpha_{\text {iso }}^{-1}$ from Eq. 10 with $S_{k_{f}}^{f}=0$, i.e. an isostatic
} 
Table 1: Material and compression parameters used for the simulation.

\begin{tabular}{|l|c|c|}
\hline Name & Symbol & Value \\
\hline Particle radius & $r$ & $2.89 \times 10^{-3} \mathrm{~m}$ \\
Young modulus & $Y$ & $5 \times 10^{6} \mathrm{~Pa}$ \\
Poisson's ratio & $v$ & 0.45 \\
Restitution coefficient & $e$ & 0.3 \\
Friction coefficient & $\mu$ & 0.5 \\
Particle mass density & $\rho_{p}$ & $2.5 \times 10^{3} \mathrm{~kg} / \mathrm{m}^{3}$ \\
\hline
\end{tabular}

Also, we kept track of the isotropic and deviatoric stress scalars $p=\frac{\sigma_{1}+\sigma_{2}+\sigma_{3}}{3}$ and $q=\sqrt{\frac{\left(\sigma_{1}-\sigma_{2}\right)^{2}+\left(\sigma_{2}-\sigma_{3}\right)^{2}+\left(\sigma_{1}-\sigma_{3}\right)^{2}}{2}}$ (with $\sigma_{1,2,3}$ are the eigenvalues of the stress tensor ${ }^{3}$ ) to assure an anisotropy ratio $\frac{q}{p}<10^{-2}$ for the limit state. The analysis was then performed on all particles at least three diameters away from the walls, and excluding rattlers. All other material properties are listed in Table 1.

Let us take $P_{\text {wall }}=55.5 \mathrm{~Pa}$ as an example. The set of all Voronoï cells follows a k-gamma distribution with $k_{v} \approx 14$ and $\chi / d^{3}=0.0146$, as in Ref.[10]. Nevertheless, not all Voronoï cells are the same. When divided into subsets by their number of faces, they still exhibit kgamma distributions, all with almost the same compactivity $\left(\chi / d^{3} \sim 0.0085(4)\right.$ for subsets with more than 1000 cells, which is different from the global one), but with different $k \mathrm{~s}$ (Fig. 1, left). Indeed, the results show a linear relation between the number of elementary cells per Voronoï cell $k_{v}$ and the number of faces $s$ of such cell, $k_{v}=2.9(1) s-19(2)^{4}$ (Fig. 1, right).
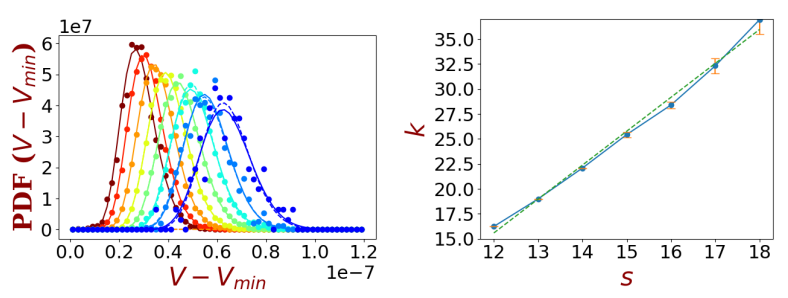

Figure 1: Distributions of Voronoï cell volumes classified by their number of faces $s$. Solid lines represent the kgamma distribution with $k_{v}$ and $\chi$ calculated from Eq. (4) while dotted lines are the least squares fitting to a k-gamma function (left). Number of elementary cells per Voronoï cell $k_{v}$ as a function of $s$ (right).

In a similar manner, the pressures per grain follows a k-gamma distribution with $k=1.78$ and $\alpha^{-1}=4.78 \times 10^{-3}$ (Fig. 2, left). When classified by the number of contacts $z$, they still show k-gammas with almost the same angoricity $\left(\alpha^{-1} \sim 0.0036\right.$ (2) for subsets with more than 1000 grains, which is different from the global one), but with different $k \mathrm{~s}$, which in the limit of low angoricity should obey Eq.

\footnotetext{
${ }^{3}$ The granular stress tensor was computed from the microscopic interactions as $\sigma_{\alpha, \beta}=\frac{1}{2 V} \sum_{i j} f_{i j, \alpha} r_{i j, \beta}$ where $\overrightarrow{r_{i j}}$ is the vector joining the centers of grains $i$ and $j$ and $\overrightarrow{f_{i j}}$ is the interaction force between them.

${ }^{4}$ Values between parentheses are 1 sigma error bars
}

(7). We observe the linear relationship $k_{f}=0.63(9) z-$ $0.5(3)$, independent of the pressure applied on the walls on the range $13.9 \mathrm{~Pa}<P_{\text {wall }}<222 \mathrm{~Pa}$ (Fig. 2, right).
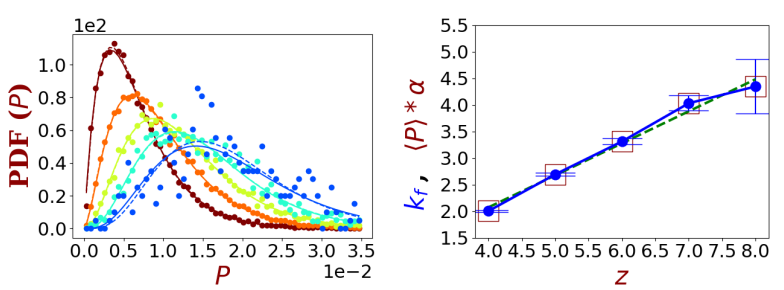

Figure 2: Distributions of the pressure per particle $p$ classified by the number of contacts $z$. Solid lines are the kgamma distributions with $k_{f}$ and $\alpha$ computed from Eq. (9), while dotted lines are least squares fittings to a k-gamma function (left). Number of elementary forces $k_{f}$ (circles) and average pressure per grain $\langle p\rangle$ times the angoricity $\alpha^{-1}$ (squares) as functions of $z$ (right).

The connection between both ensembles is established by linking the number of faces $s$ for the Voronoï cells and the number of contacts $z$ for the particles. The joint distribution of $s$ and $z$ is almost the same for all $P_{\text {wall }}$ on the established range (Fig. 3,left). Although a particle with a given number of contacts can have a Voronoï cell with different number of faces (and vice-versa), for every $z$ there is a preference for a given $s$. Indeed their averages follow linear relations (Fig. 3, right). We propose $s=-0.30(1) z+15.9(9)$, where the negative sign implies that adding contacts reduces the number of faces.
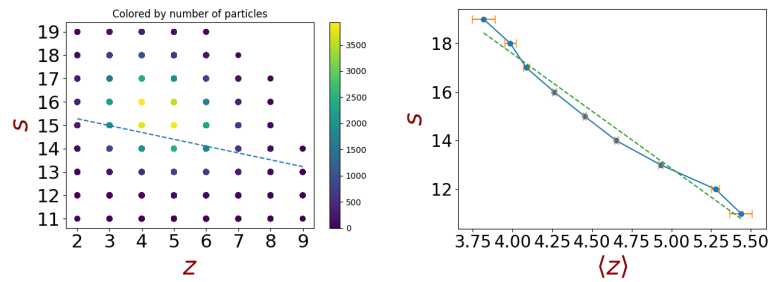

$\langle z\rangle$

Figure 3: Distribution of grains according to the number of faces of their Voronoï cell $s$ and their number of contacts $z$ (left). Relation between the number of faces $s$ of the Voronoï cell and the average number of contacts $\langle z\rangle$ (right).

By assuming that pressure and volume are uncorrelated variables (a reasonable assumption for very rigid spheres), the total entropy $S_{\text {Total }}$ will be the sum of those for the volume and the force network ensembles (Eq. $(2,10))$,

$$
\frac{S_{\text {Total }}}{N}=\frac{C}{N}\left[1+\ln \left(\frac{\chi}{\Lambda^{3}}\right)\right]+\frac{k_{f}}{N}\left[1+\ln \left(\frac{\alpha^{-1}}{p_{0}}\right)\right],
$$

where $C / N=k_{v}$ is the number of elementary cells per Voronoï cell and $k_{f} / N=k_{f}^{\prime}$ is the number of force degrees of freedom per particle. Using the linear expressions before, the parameters $k_{v}$ and $k_{f}$ can be written as function of the number of contacts: $k_{v}=-M_{v} z+B$ and $k_{f}=M_{f} z-D$, 
with $\left.M_{v}=0.87(8), B=27(7), M_{f}=0.63(9)\right)$ and $D=0.5(3)$. Then, the total entropy can be written as a function of the coordination number $z$,

$$
\frac{S_{\text {Total }}}{N}=\left(-M_{v} z+B\right)\left[1+\ln \left(\frac{\chi}{\Lambda^{3}}\right)\right]+\left(M_{f} z-D\right)\left[1+\ln \left(\frac{\alpha^{-1}}{p_{0}}\right)\right] .
$$

The force entropy grows with the number of contacts, while the volume entropy decreases; therefore, in a statistical equilibrium state we could expect entropy to remain almost constant (i.e. maximized) with the number of contacts. Figure 4 (left) shows that this is indeed the case, with a total variation around $3 \%$ for the five values of $P_{\text {wall }}$.
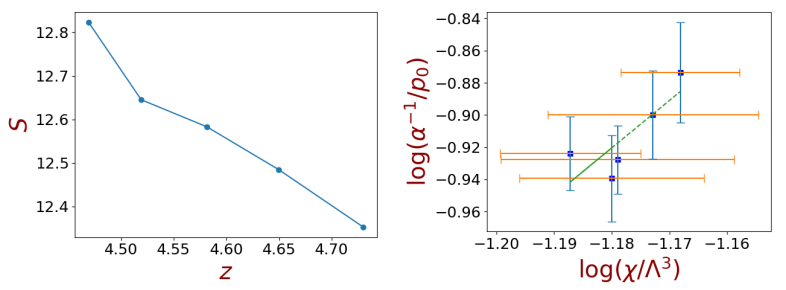

Figure 4: Entropy per grain as a function of the average number of contacts of the sample, Eq. (13) (left). Relation between the two terms in the proposed equation of state (Eq. 14) (right). The slope in the linear fit is $2.94 \pm 1.21$.

Finally, maximizing the total entropy against the number of contacts $z$,

$$
\frac{\partial S_{\mathrm{Total}} / N}{\partial z}=M_{f}\left[1+\ln \left(\frac{\alpha^{-1}}{p_{0}}\right)\right]-M_{v}\left[1+\ln \left(\frac{\chi}{\Lambda^{3}}\right)\right]=0,
$$

gives an expression relating angoricity and compactivity,

$$
\frac{\alpha^{-1}}{p_{0}}=e^{\frac{M_{v}}{M_{f}}-1}\left(\frac{\chi}{\Lambda^{3}}\right)^{M_{v} / M_{f}}
$$

that is, an equation of state for a granular medium of rigid monodisperse spheres under low pressure isotropic compressions. Fig. 4 (right), shows that the logarithms of the angoricities for the subsets classified by $z$ and the logarithms of the compactivities for the subsets classified by $s$ fulfill a linear relation, as expected from Eq. (14). The positive slope of $2.94 \pm 1.21$ is close enough to the expected value $M_{v} / M_{f}=1.38$ to encourage us for developing more research in the future.

\section{Conclusions}

Summarizing, this work proposes a procedure to join the force network ensemble on a single grain and the volume ensemble of the Voronoï cell around it for a monodisperse spherical granular medium in the limit of isotropic compression, just by establishing an empirical relation between the number on contacts on the grain with the number of faces of the Voronoï cell. Using this relation we express the total entropy of the system as a function of the number of contacts, which is expected to be approximately constant in equilibrium. From there we propose a state equation between the average pressure per particle and the packing fraction, to be verified in future works. The procedure constitutes a further step to reach the holy grail of granular media, i.e. to develop a comprehensive statistical theory of granular media joining the volume ensemble and the force networks, clearing a way towards a theoretical description of dense granular media in static equilibrium.

\section{References}

[1] B. Andreotti, Y. Forterre, O. Pouliquen, Granular Media: Between Fluid and Solid (Cambridge University Press, 2013)

[2] A.J. Liu, S.R. Nagel, Nature 396, 21 (1998)

[3] S. Edwards, R. Oakeshott, Physica A 157, 1080 (1989)

[4] A. Baule, F. Morone, H.J. Herrmann, H.A. Makse, Rev. Mod. Phys. 90, 015006 (2018)

[5] B. Tighe, T. Vlugt, J. Stat. Mech-Theory E 2011, P04002 (2011)

[6] B. Tighe, J. Snoeijer, T. Vlugt, M. van Hecke, Soft matter 6, 2908 (2010)

[7] J. Snoeijer, T. Vlugt, W. Ellenbroek, M. Hecke, J. Leeuwen, Phys. Rev. E 70, 061306 (2005)

[8] R.C. Ball, R. Blumenfeld, Phys. Rev. Lett. 88, 115505 (2002)

[9] T. Aste, T.D. Matteo, M. Saadatfar, T.J. Senden, M. Schröter, H.L. Swinney, Europhys. Lett. 79, 24003 (2007)

[10] W.F. Oquendo, J.D. Muñoz, F. Radjai, EPLEurophys. Lett. 114, 14004 (2016)

[11] P. Moschopoulos, Ann. I. Stat. Math. 37, 541 (1985)

[12] T. Aste, T. Di Matteo, Phys. Rev. E 77, 021309 (2008)

[13] R. Kurita, E.R. Weeks, Phys. Rev. E 82, 011403 (2010)

[14] J. Kepler, The Six-Cornered Snowflake (Oxford: Clarendon, 1611)

[15] T.C. Hales, Ann.Math. 162, 1065-1185 (2005)

[16] F. Radjai, S. Roux, J.J. Moreau, Chaos 9, 544 (1999)

[17] P.T. Metzger, C.M. Donahue, Phys. Rev. Lett. 94, 148001 (2005)

[18] B.P. Tighe, T.J.H. Vlugt, J. Stat. Mech-Theory E. 2010, P01015 (2010)

[19] M. Cárdenas-Barrantes, J. Muñoz, W. Oquendo, Granul. Matter 20 (2017)

[20] C. Kloss, C. Goniva, A. Hager, S. Amberger, S. Pirker, Prog. Comput. Fluid Dy. 12, 140 (2012)

[21] T. Pöschel, T. Schwager, Computational Granular Dynamics: Models and Algorithms (Springer-Verlag, 2005), ISBN 978-3-540-21485-4.

[22] D. Frenkel, B. Smit, Understanding molecular simulation : from algorithms to applications. 2nd ed, Vol. 50 (Academic Press, 1996)

[23] B.D. Lubachevsky, F.H. Stillinger, J. Stat. Phys. 60, 561 (1990) 